\title{
Adjustment of Turbulent Boundary-Layer Flow to Idealized Urban Surfaces: A Large-Eddy Simulation Study
}

\author{
Wai-Chi Cheng · Fernando Porté-Agel
}

Received: 25 July 2014 / Accepted: 5 January 2015 / Published online: 28 January 2015

C Springer Science+Business Media Dordrecht 2015

\begin{abstract}
Large-eddy simulations (LES) are performed to simulate the atmospheric boundary-layer (ABL) flow through idealized urban canopies represented by uniform arrays of cubes in order to better understand atmospheric flow over rural-to-urban surface transitions. The LES framework is first validated with wind-tunnel experimental data. Good agreement between the simulation results and the experimental data are found for the vertical and spanwise profiles of the mean velocities and velocity standard deviations at different streamwise locations. Next, the model is used to simulate ABL flows over surface transitions from a flat homogeneous terrain to aligned and staggered arrays of cubes with height $h$. For both configurations, five different frontal area densities $\left(\lambda_{f}\right)$, equal to $0.028,0.063,0.111$, 0.174 and 0.250 , are considered. Within the arrays, the flow is found to adjust quickly and shows similar structure to the wake of the cubes after the second row of cubes. An internal boundary layer is identified above the cube arrays and found to have a similar depth in all different cases. At a downstream location where the flow immediately above the cube array is already adjusted to the surface, the spatially-averaged velocity is found to have a logarithmic profile in the vertical. The values of the displacement height are found to be quite insensitive to the canopy layout (aligned vs. staggered) and increase roughly from $0.65 h$ to $0.9 h$ as $\lambda_{\mathrm{f}}$ increases from 0.028 to 0.25 . Relatively larger values of the aerodynamic roughness length $\left(z_{0}\right)$ are obtained for the staggered arrays, compared with the aligned cases, and a maximum value of $z_{0}$ is found at $\lambda_{\mathrm{f}}=0.111$ for both configurations. By explicitly calculating the drag exerted by the cubes on the flow and the drag coefficients of the cubes using our LES results, and comparing the results with existing theoretical expressions, we show that the larger values of $z_{0}$ for the staggered arrays are related to the relatively larger drag coefficients of the cubes for that configuration compared with the aligned one. The effective mixing length $\left(l_{\mathrm{m}}\right)$ within and above different cube arrays is also calculated and a local maximum of $l_{\mathrm{m}}$ within the canopy is found in all the cases, with values ranging from $0.2 h$ to $0.4 h$. These patterns of $l_{\mathrm{m}}$ are different from those used in existing urban canopy models.
\end{abstract}

W.-C. Cheng · F. Porté-Agel ( $\square)$

Wind Engineering and Renewable Energy Laboratory (WIRE), École Polytechnique Fédérale de Lausanne (EPFL), Lausanne, Switzerland

e-mail: fernando.porte-agel@epfl.ch 
Keywords Flow adjustment - Large-eddy simulation - Model validation - Uniform cube arrays · Urban canopy flows

\section{Introduction}

Due to the increasing concerns about air quality in urban areas, many recent studies have aimed to investigate the complex interactions between turbulent atmospheric boundary-layer (ABL) flow and the underlying cities. The main objective was to characterize the turbulent flow structure, quantify the turbulent fluxes of momentum and scalars, and develop simple parametrizations of those fluxes that can be used for the prediction of air quality and airflow patterns in urban environments.

In the literature, there are numerous wind-tunnel studies (Raupach et al. 1980; Brown et al. 2001; Macdonald et al. 2002; Castro et al. 2006), large-eddy simulations (LES) (Kanda 2006; Xie et al. 2008; Boppana et al. 2010; Castillo et al. 2011) and direct numerical simulations (DNS) (Coceal et al. 2006, 2007a, b) of flows above idealized building geometries. The goals of many of these studies are to investigate and to characterize the mean wind and turbulence within and above urban canopies. One important focus is the characterization of the surfaces using simple bulk parameters that can be used in surface-layer similarity theory, e.g. MoninObukhov similarity (Businger et al. 1971). Of particular interest is the determination of the effective aerodynamic roughness length $\left(z_{0}\right)$ and displacement height $(d)$ that are key parameters in the logarithmic wind law,

$$
\frac{U(z)}{u_{*}}=\frac{1}{\kappa} \ln \left(\frac{z-d}{z_{0}}\right),
$$

where $U$ is the temporally- and spatially-averaged wind speed, $z$ is the height, $u_{*}$ is the friction velocity and $\kappa$ is the von Kármán constant. Traditionally, $z_{0}$ and $d$ are usually expressed as functions of the roughness-element density $(\lambda)$ as summarized in Grimmond and Oke (1999). Commonly used parameters to represent $\lambda$ include the frontal area density $\left(\lambda_{\mathrm{f}}=A_{\mathrm{f}} / A_{\mathrm{d}}\right.$ ) and the plan area density $\left(\lambda_{\mathrm{p}}=A_{\mathrm{p}} / A_{\mathrm{d}}\right)$, where $A_{\mathrm{f}}$ is the total frontal area of the obstacles, $A_{\mathrm{p}}$ is the total plan area of the obstacles and $A_{\mathrm{d}}$ is the total area covered by obstacles. Previous wind-tunnel experiments (as reviewed in Raupach 1992; Macdonald et al. 1998 and Grimmond and Oke 1999) generally showed that $d$ increases with $\lambda$ while $z_{0}$ shows a maximum value at $\lambda \approx 0.1-0.2$. By considering the sheltering effects of the roughness elements of an array of obstacles, Raupach $(1992,1994)$ proposed an analytic treatment of the drag force and drag partition and suggested expressions for $z_{0}$ and $d$ as functions of $\lambda$. Later, Macdonald et al. (1998) proposed other simple expressions for $z_{0}$ and $d$, also as functions of $\lambda$, by considering the drag force exerted by the roughness elements on the flow. However, in Raupach (1992, 1994) and Macdonald et al. (1998), the effects of roughness-element configuration and atmospheric conditions, which are also important parameters affecting the values of $z_{0}$ and $d$, were not considered. For $z_{0}$ and $d$ estimated from the wind profiles, there was also the problem that different estimated values were obtained using different methods (Petersen 1997; Macdonald 2000; Cheng and Castro 2002; Cheng et al. 2007).

In their wind-tunnel experiment, Raupach et al. (1980) considered flow above regular arrays of wooden cylinders with five different densities to investigate how wake diffusion and horizontal inhomogeneity affect the mean wind profiles above the surfaces. In other wind-tunnel studies, Cheng and Castro (2002) investigated the horizontal heterogeneity of the flow above arrays of blocks and tried to identify the inertial (surface) layer and roughness sublayer. They found that the inertial-layer depths are the same for elements with uniform 
and random height, while a much thicker roughness sublayer was found for the random surface. They also found that the dispersive stresses are negligible compared with the turbulent Reynolds stresses. Later, Castro et al. (2006) used experimental data of flow over a staggered cube array in neutral conditions to analyze the velocity spatial correlation, velocity spectra, quadrants of the turbulent shear stress, and the turbulent kinetic energy (TKE) balance. The experiments of Cheng and Castro (2002) and Castro et al. (2006) also provided useful data for validating computational fluid dynamics (CFD) models, e.g. the DNS of Coceal et al. (2006, 2007a,b). Coceal et al. (2006, 2007a,b) were able to calculate different turbulence statistics and analyze the coherent structure and turbulent length scale within and above an array of cubes. Kanda (2006) used LES to study the turbulence structure above surfaces of block arrays with different densities and block height arrangements. In addition to the experimental and numerical studies mentioned above, there were also studies (Macdonald 2000; Coceal and Belcher 2004, 2005) that aimed to develop simple analytical models for urban canopy flows based on the Prandtl mixing-length theory and the drag exerted by the buildings on the flow.

For the studies mentioned above, fully adjusted flows above urban-like surfaces were considered. In comparison, few studies have been conducted to investigate the ABL transition for flow over a city. These include the wind-tunnel studies of Cheng and Castro (2002) and Schultz et al. (2005) that investigated the flows over smooth-to-rough surface transitions. Cheng and Castro (2002) considered a rough surface with roughness elements of height about $1 \%$ of the boundary-layer depth. By considering the vertical profiles of mean velocity and turbulent characteristics, they found that the flow in the roughness sublayer is adjusted to the surface after a distance equal to about 160 times the aerodynamic roughness length of the downstream surface. They also tried to identify the growing equilibrium layer and roughness sublayer, which were found to be sensitive to the way they are defined. On the other hand, Schultz et al. (2005) used roughness elements with a height of about $2.5 \%$ of the boundary-layer depth to investigate the adjustment of the flow. They found that the flow reaches equilibrium more rapidly when a more turbulent approaching flow is used. The analytical studies of Belcher et al. (2003) introduced the canopy-drag length scale $\left(L_{\mathrm{c}}\right)$ as a fundamental dynamical length scale of urban canopies. Coceal and Belcher (2004) used $L_{\mathrm{c}}$ to estimate the distance for flow adjustment as a flow enters an urban canopy.

The objective of this study is to investigate the flow characteristics of an ABL flow as it passes uniform arrays of cubes using LES. LES can reproduce the most energetic turbulence motions and has the advantage of relatively low computational cost compared with DNS (e.g., Meneveau and Katz 2000; Pope 2000; Voller and Porté-Agel 2002; Sagaut 2006). This allows the use of a relatively large computational domain that is essential in reproducing the large-scale turbulence above cities and ensuring there is enough space for flow adjustment after the flow passes a surface transition. In Sect. 2, the LES framework is introduced. The code is first validated with the wind-tunnel experimental data of Brown et al. (2001) and the results are shown in Sect. 3. In Sect. 4, the results obtained from the LES of ABL flows over surface transitions from a flat homogeneous terrain to aligned and staggered cube arrays with different densities are presented and discussed. Finally, in Sect. 5, the main results are summarized and conclusions drawn.

\section{LES Framework}

The LES code solves the filtered continuity equation and the filtered incompressible NavierStokes equations in rotational form (Orszag and Pao 1975), 


$$
\begin{aligned}
\frac{\partial \tilde{u}_{i}}{\partial x_{i}} & =0, \\
\frac{\partial \tilde{u}_{i}}{\partial t}+\tilde{u}_{j}\left(\frac{\partial \tilde{u}_{i}}{\partial x_{j}}-\frac{\partial \tilde{u}_{j}}{\partial x_{i}}\right) & =-\frac{\partial \tilde{p}^{*}}{\partial x_{i}}-\frac{\partial \tau_{i j}}{\partial x_{j}}+v \frac{\partial^{2} \tilde{u}_{i}}{\partial x_{j}^{2}}+f_{i}+F_{i},
\end{aligned}
$$

where $\tilde{u}_{i}$ is the filtered velocity component in the $i$ direction (with $i=1,2,3$ corresponding to the streamwise $(x)$, spanwise $(y)$ and vertical $(z)$ direction, respectively), $\tilde{p}^{*}=\tilde{p} / \rho+\frac{1}{2} \tilde{u}_{i} \tilde{u}_{i}$ is the modified kinematic pressure, $\tilde{p}$ is the filtered pressure, $\rho$ is the density of air, $\tau_{i j}=$ $\widetilde{u_{i} u_{j}}-\tilde{u_{i}} \tilde{u_{j}}$ is the subgrid-scale (SGS) stress tensor, and $v$ is the kinematic viscosity of air. The term $f_{i}$ is the immersed forcing used to simulate the effects of the blocks on the flow and $F_{i}$ is a mean pressure gradient forcing term. Neutrally stratified conditions are considered and therefore no additional term is required to account for the effect of buoyancy.

The SGS model used here is the modulated gradient model of Lu and Porté-Agel (2010). The SGS stress is calculated as

$$
\tau_{i j}=2 k_{\mathrm{sgs}}\left(\frac{\widetilde{G}_{i j}}{\widetilde{G}_{k k}}\right),
$$

where $k_{\mathrm{sgs}}$ is the SGS kinetic energy and $\widetilde{G}_{i j}$ is obtained by the Taylor's expansion of $\tau_{i j}$ as

$$
\widetilde{G}_{i j}=\frac{\Delta_{x}^{2}}{12} \frac{\partial \tilde{u}_{i}}{\partial x} \frac{\partial \tilde{u}_{j}}{\partial x}+\frac{\Delta_{y}^{2}}{12} \frac{\partial \tilde{u}_{i}}{\partial y} \frac{\partial \tilde{u}_{j}}{\partial y}+\frac{\Delta_{z}^{2}}{12} \frac{\partial \tilde{u}_{i}}{\partial z} \frac{\partial \tilde{u}_{j}}{\partial z},
$$

where $\Delta_{x}, \Delta_{y}$ and $\Delta_{z}$ correspond to the streamwise, spanwise and vertical grid spacings, respectively. The value of $k_{\mathrm{sgs}}$ is calculated with the assumption of local equilibrium. To ensure numerical stability, no local energy transfer from unresolved to resolved scales is allowed (Lu and Porté-Agel 2010). Thus,

$$
k_{\mathrm{sgs}}= \begin{cases}\frac{4 \Delta^{2}}{C_{\epsilon}^{2}}\left(-\frac{\widetilde{G}_{i j}}{\widetilde{G}_{k k}} \widetilde{S}_{i j}\right)^{2} & \text { if } \widetilde{G}_{i j} \widetilde{S}_{i j}<0, \\ 0 & \text { otherwise },\end{cases}
$$

with $\Delta=\left(\Delta_{x} \Delta_{y} \Delta_{z}\right)^{1 / 3}, \widetilde{S}_{i j}=\frac{1}{2}\left(\partial \tilde{u}_{i} / \partial x_{j}+\partial \tilde{u}_{j} / \partial x_{i}\right)$ is the resolved strain-rate tensor, and $C_{\epsilon} \approx 1$ based on the assumption of an averaged energy balance between SGS energy production and molecular dissipation rate. The main advantages of the modulated gradient model are: (i) the model satisfies material frame indifference, (ii) it is simple and computationally inexpensive as neither test filtering nor additional transport equation is needed, and (iii) it is able to capture flow anisotropy better than eddy-viscosity models.

In all the simulations, the top boundary was considered as a stress-free wall. Periodic boundary conditions were used in the lateral directions with a buffer zone to impose a boundary-layer inflow condition. To do so, a precursor simulation technique was adopted, and involved running a prior simulation of the turbulent boundary-layer flow without cubes. The instantaneous velocity fields obtained from the precursor simulation were saved and later used as inflows to the simulations of flow past cube arrays, using a buffer zone to adjust the flow from the downwind condition to that of the precursor simulation. The use of this precursor simulation technique to impose the inflow condition while maintaining the accuracy in a similar pseudospectral LES codes has been successful in previous studies of flow past a 2D block (Cheng and Porté-Agel 2013), an urban canopy (Tseng et al. 2006), a steep hill (Wan and Porté-Agel 2011), a wind turbine (Porté-Agel et al. 2011; Wu and Porté-Agel 2011, 2012), and wind farms (Porté-Agel et al. 2013; Wu and Porté-Agel 2013). For the bottom wall, the boundary condition was based on the Monin-Obukhov similarity theory (Businger 
et al. 1971). Although the theory is only valid for averaged quantities under steady and homogeneous conditions, it is commonly used also for fluctuating (LES filtered) quantities in both homogeneous and heterogeneous flows. Even though recent studies have highlighted the limitations of this approach (e.g., Marusic et al. 2001; Chamorro and Porté-Agel 2010; Abkar and Porté-Agel 2012), no alternative is available for complex flows. Under neutral conditions, the surface shear stress $\tau_{i 3, s}(x, y, t)(i=1,2)$ is often computed as (Stoll and Porté-Agel 2006, 2008)

$$
\tau_{i 3, s}(x, y, t)=-\left[\frac{\kappa \tilde{u}_{r}(x, y, z, t)}{\ln \left(z / z_{0}\right)}\right]^{2} \frac{\tilde{u}_{i}(x, y, z, t)}{\tilde{u}_{r}(x, y, z, t)},
$$

where the subscript $s$ denotes surface values, $t$ is the time, $\tilde{u}_{r}(x, y, z, t)=\left[\tilde{u}_{1}(x, y, z, t)^{2}+\right.$ $\left.\tilde{u}_{2}(x, y, z, t)^{2}\right]^{1 / 2}$ is the local instantaneous (filtered) horizontal velocity magnitude at height $z=\Delta_{z} / 2$, and $z_{0}$ is the aerodynamic roughness length.

In the simulations, the spatial derivatives in the horizontal directions were calculated based on the pseudo-spectral method, while a second-order central-difference method was used in the vertical direction. The time advancement was carried out using the second-order Adams-Bashforth scheme (Canuto et al. 1988). More details on the LES code can be found in Albertson and Parlange (1999), Porté-Agel et al. (2000, 2011), Porté-Agel (2004), Stoll and Porté-Agel (2006, 2008) and Lu and Porté-Agel (2010).

An immersed boundary method (Mittal and Iaccarino 2005; Cheng and Porté-Agel 2013) was used to model the presence of blocks by specifying an immersed forcing term $f_{i}$ in Eq. 3. A Cartesian grid with staggered arrangement was used. The levels of computation for the vertical velocity component $\tilde{w}$ were half-grid shifted compared with the computational levels for the streamwise velocity component $\tilde{u}$, spanwise velocity component $\tilde{v}$, and $\tilde{p}^{*}$. The vertical surfaces of the blocks were located at a position aligned with a grid column, while the top surface was located at the computational level of $\tilde{w}$. At the block surfaces, the surface shear stress was computed using a method similar to that for the bottom surface. This required the calculation of the instantaneous (filtered) surface shear stress as a function of the velocity field at the first grid point outside the surfaces. The boundary condition was then accomplished through the local application of Monin-Obukhov similarity theory. Moreover, smoothing of the velocity field inside the blocks before the velocity derivative calculations, was used to diminish the Gibbs phenomenon that occurs near sharp boundaries (Tseng et al. 2006; Fang et al. 2011).

In the following discussion of the LES results, the filtered velocity $\tilde{u}_{i}$ is decomposed into the time average $\left\langle\tilde{u}_{i}\right\rangle$ and fluctuation $\tilde{u}_{i}^{\prime}$ components as

$$
\tilde{u}_{i}=\left\langle\tilde{u}_{i}\right\rangle+\tilde{u}_{i}^{\prime},
$$

where the statistics are computed after the flow achieves quasi-steady conditions. In addition, the streamwise, spanwise and vertical velocity components are sometimes denoted as $u, v$ and $w$, respectively.

\section{LES Validation}

The LES framework was validated in our previous study (Cheng and Porté-Agel 2013) in simulations of a turbulent boundary-layer flow past a 2D block. In order to extend the LES validation to flows over three-dimensional urban surfaces, here we evaluate the LES framework against the wind-tunnel experimental data of Brown et al. (2001) for turbulent 
(a)

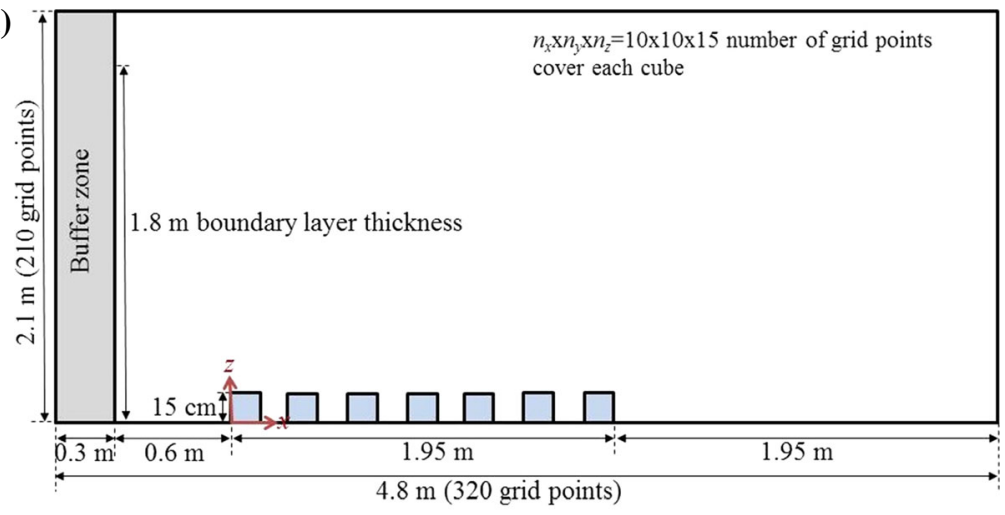

(b)

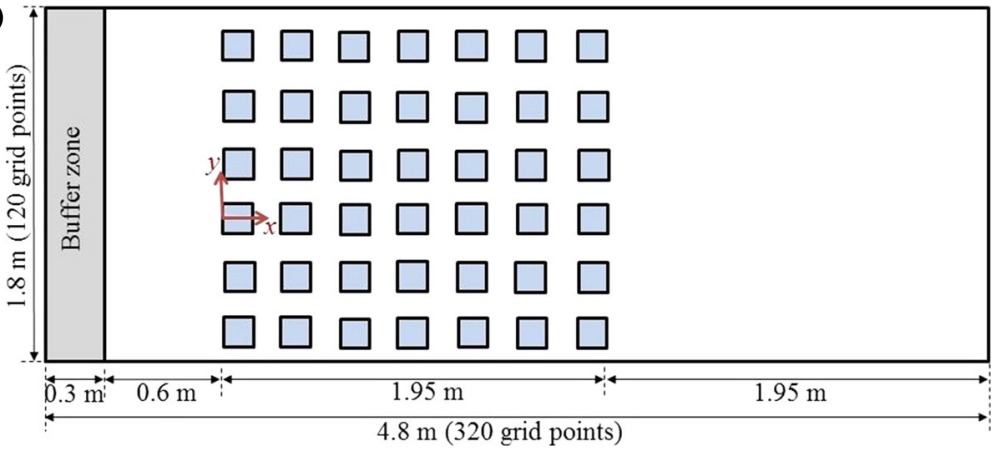

Fig. 1 Computational domain for model validation case: a cross-sectional view, and $\mathbf{b}$ top view

boundary-layer flow past an aligned array of cubes. The experiment was carried out in the U.S Environmental Protection Agency's Fluid Modeling Facility wind tunnel. The test section of the wind tunnel was $3.7 \mathrm{~m}$ wide, $2.1 \mathrm{~m}$ high and $18.3 \mathrm{~m}$ long, with the aligned array consisting of $7 \times 11$ cubes with height $h=0.15 \mathrm{~m}$ and spacings between cubes equal to $h$ in both streamwise and spanwise directions. A neutral turbulent boundary layer with a depth of $1.8 \mathrm{~m}$ was produced using spires and floor roughness elements (with $z_{0}=1 \mathrm{~mm}$ ). The Reynolds number $(R e)$ of the experiment, based on the cube height $h$ and the velocity at $z=h$, was approximately equal to $3 \times 10^{4}$. Pulsed-wire anemometry (PWA) was used to measure the velocity time series.

The computational domain, shown in Fig. 1, had dimensions $L_{x} \times L_{y} \times L_{z}$ equal to $4.8 \mathrm{~m} \times 1.8 \mathrm{~m} \times 2.1 \mathrm{~m}$, the domain was divided into $N_{x} \times N_{y} \times N_{z}=320 \times 120 \times 210$ nodes. This corresponded to a number of grid points covering each cube of $n_{x} \times n_{y} \times n_{z}=$ $10 \times 10 \times 15$. The value of $z_{0}$ for the upstream ground was equal to $1 \mathrm{~mm}$. For the ground within the cube array and the walls of the cubes, $z_{0}=0.01 \mathrm{~mm}$ was used based on the relation $z_{0}=0.12 v / u_{*}$ for aerodynamically smooth surfaces (Hinze 1975). The height of the domain $L_{z}$ was slightly higher than the depth of the incoming boundary layer $\delta(=1.8$ $\mathrm{m})$ to allow for possible boundary-layer growth. A buffer zone, located at a distance of 0.6 $\mathrm{m}$ upstream of the cube array, was used to impose the inflow condition using the precursor simulation technique described in Sect. 2. A precursor simulation of a turbulent boundarylayer flow without cubes was performed to generate the inflow condition. The generated mean flow was characterized by a logarithmic profile with $z_{0}=1 \mathrm{~mm}$ and $u_{*}=0.22 \mathrm{~m} \mathrm{~s}^{-1}$. The profiles of the normalized mean streamwise velocity component $\left(\langle\tilde{u}\rangle / u_{*}\right)$ and velocity 

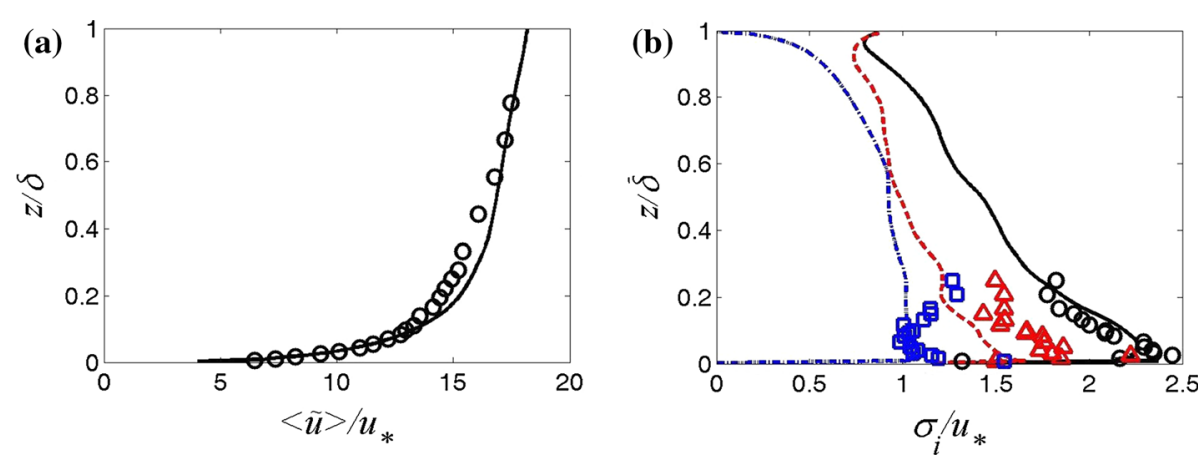

Fig. 2 Vertical profiles of a normalized mean streamwise velocity component $\left(\langle\tilde{u}\rangle / u_{*}\right)$ and $\mathbf{b}$ normalized velocity standard deviations $\left[\sigma_{u} / u_{*}\right.$ (black line and circle symbol), $\sigma_{v} / u_{*}$ (red dashed line and triangle) and $\sigma_{w} / u_{*}$ (blue dashed dot line and square)] simulated by precursor simulation. Symbols experimental data; lines current LES results

standard deviations $\left(\sigma_{i} / u_{*}\right)$ simulated by the precursor simulation are shown in Fig. 2 and compared with the experimental profiles measured at $500 \mathrm{~mm}(3.3 \mathrm{~h})$ upstream of the cube array.

The comparison between the LES results and the wind-tunnel measurements of the normalized mean velocity components $\left(\langle\tilde{u}\rangle / U_{0}\right.$ and $\left.\langle\tilde{w}\rangle / U_{0}\right)$ and velocity standard derivations $\left(\sigma_{u} / U_{0}, \sigma_{v} / U_{0}\right.$ and $\left.\sigma_{w} / U_{0}\right)$ at different streamwise locations of the cube array are shown in Figs. 3 and 4, respectively. $U_{0}$ is the freestream mean wind speed. For the vertical and spanwise profiles of $\langle\tilde{u}\rangle$ and $\langle\tilde{w}\rangle$, good agreement is observed between the simulation results and the experimental data. This demonstrates that the model is able to reproduce accurately the flow recirculation pattern within and around the cube array. Moreover, good predictions of the velocity standard deviations $\left(\sigma_{u} / U_{0}, \sigma_{v} / U_{0}\right.$ and $\left.\sigma_{w} / U_{0}\right)$ by the current simulation are also observed in the vertical and spanwise profiles shown in Fig. 4. The magnitudes of the velocity standard deviations are well reproduced by the simulation at all locations except at the positions close to the top walls of the first row of cubes. At those positions, smaller magnitudes of the velocity standard deviations are predicted. The difference observed is possibly due to the very sharp velocity change there, which causes the velocity profile to deviate significantly from the logarithmic wind profile. This, as a result, causes errors when applying the wall boundary condition (which is based on the logarithmic profile) there. However, as discussed in Sect. 2, although there are limitations to the current approach, no alternative is available yet for complex flows. In general, the current LES shows remarkable performance in simulating the mean wind and turbulence both within and above the array of cubes.

\section{LES of Atmospheric Boundary-Layer Flows Through Idealized Urban Canopies}

Here, we present results from LES of ABL flows over surface transitions from a flat homogeneous terrain to idealized urban-like surfaces with different building configurations and densities. The computational domain is shown in Fig. 5 and the idealized urban-like surfaces consist of aligned and staggered arrays of cubes (of height $h$ ) with different frontal area densities $\left(\lambda_{\mathrm{f}}\right)$ equal to $0.028,0.063,0.111,0.174$ and 0.250 . For all the cases, the dimensions $L_{x} \times L_{y} \times L_{z}$ of the computational domain were equal to $49 h \times 12 h \times 13 h$ and the domain was divided into $N_{x} \times N_{y} \times N_{z}=490 \times 120 \times 196$ nodes. This corresponded to a number 

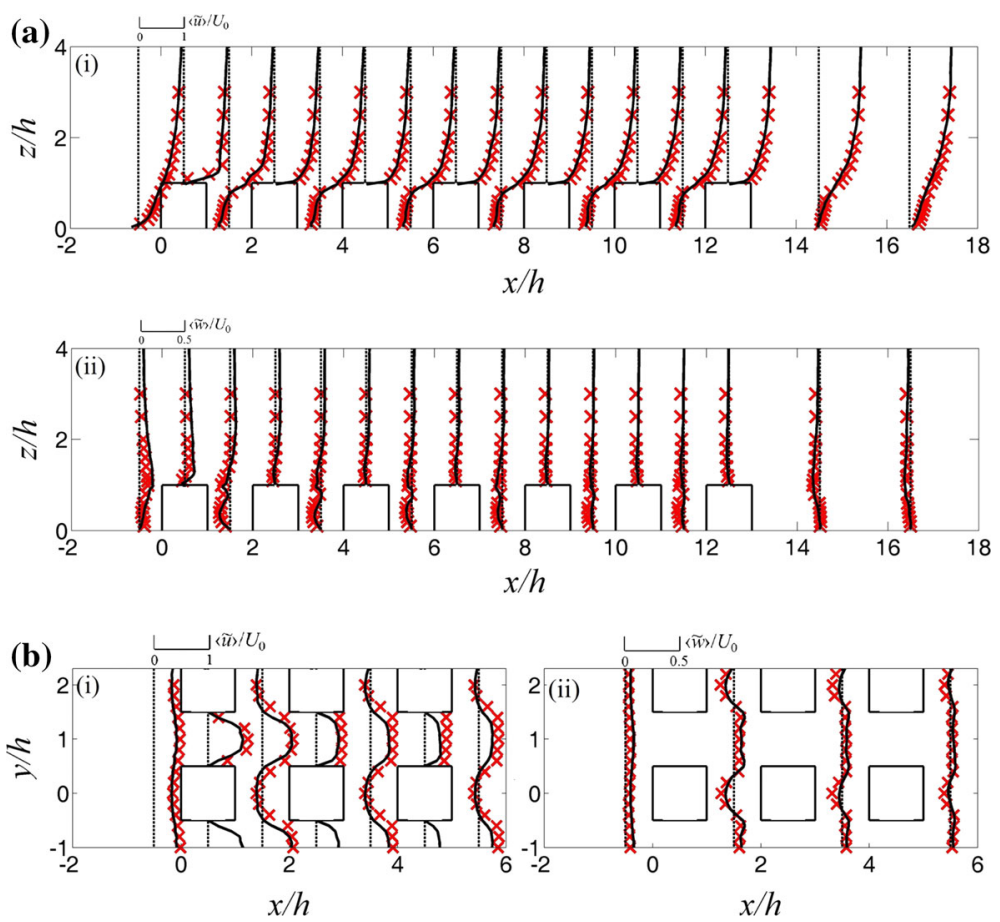

Fig. 3 a Vertical profiles at centreline of the cube array and $\mathbf{b}$ spanwise profiles at $z=h / 2$ of $(i)\langle\tilde{u}\rangle / U_{0}$ and (ii) $\langle\tilde{w}\rangle / U_{0}$. Red crosses experimental data; black lines current LES results; black dashed line $y$-axes for the profiles at different streamwise positions

of grid points covering each cube of $n_{x} \times n_{y} \times n_{z}=10 \times 10 \times 15$. The values of $z_{0}$ of the ground surface and the surfaces of the cubes were the same and equal to $0.0067 \mathrm{~h}$. Similar to the validation case discussed in Sect. 3, a precursor simulation was used to generate the inflow condition of a turbulent boundary-layer flow, of depth $12 \mathrm{~h}$, over a homogeneous flat surface with $z_{0}=0.0067 h$. A buffer zone located at a distance of $6 h$ upstream of the cube arrays was used to impose the inflow condition in the simulations.

\subsection{Flow Patterns}

The contours of the streamwise velocity component at the cube-array centreline and the edge of the internal boundary layer (IBL) for the different cases are shown in Fig. 6. The edge of the IBL is defined as the height at which the spanwise-averaged streamwise velocity component is equal to $99 \%$ of the upstream velocity. As the turbulent boundary-layer flow passes the cube arrays, the flow within the array (at heights below $h$ ) is decelerated by the drag force exerted by the cubes, while a small speed-up is found just above the first row of cubes. The flow is then gradually adjusted to the surface, and a new IBL develops above the cube array. No significant difference in the depth of the IBL among different cases is observed. These results are different from the IBL height predictions of the analytical models proposed in the literature (see Savelyev and Taylor 2005). For example, in the predictions of the models proposed by Wood (1982), Pendergrass and Aria (1984), and Savelyev and Taylor (2005), clearer dependences of the IBL thickness on canopy densities are found compared with 

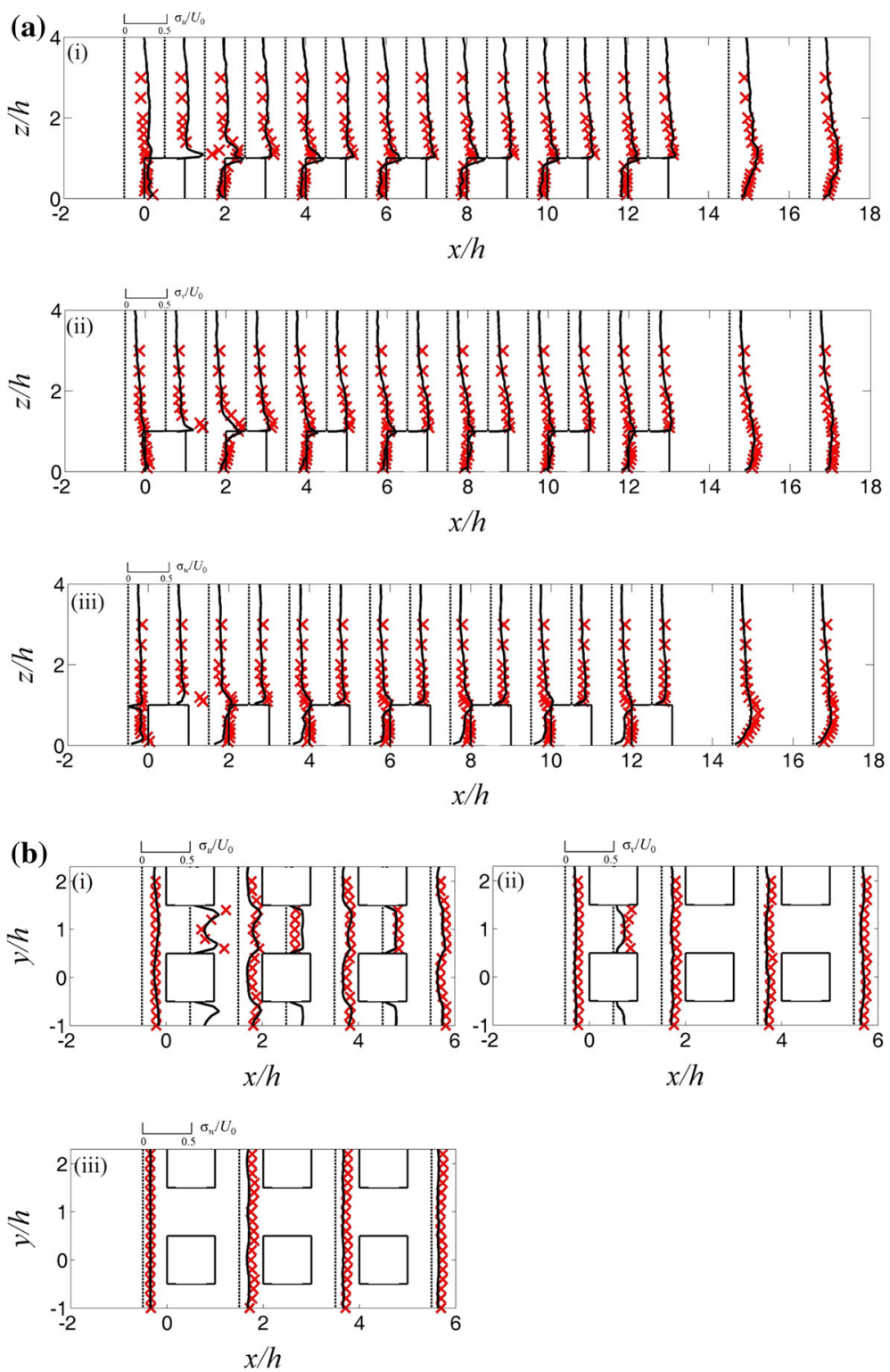

Fig. 4 a Vertical profiles at centreline of the cube array and $\mathbf{b}$ spanwise profiles at $z=h / 2$ of $(i) \sigma_{u} / U_{0}$, (ii) $\sigma_{v} / U_{0}$ and (iii) $\sigma_{w} / U_{0}$. Red crosses experimental data; black lines current LES results; black dashed line $y$-axes for the profiles at different streamwise positions

those reported in the LES results. Also, in the current LES results, the growth rate of the IBL thickness is found to decrease with streamwise position in all the cases tested. This is again different from the results of the models, which predict the IBL thickness increases almost linearly far downstream of a surface transition. The likely cause is that most of the existing IBL relations are only effective for IBLs that are located within the surface layer (with height $\approx 0.1-0.2 \delta$ ). However, in the current study, the IBL layer attains a height of $2.5 \mathrm{~h}$ 
(a)

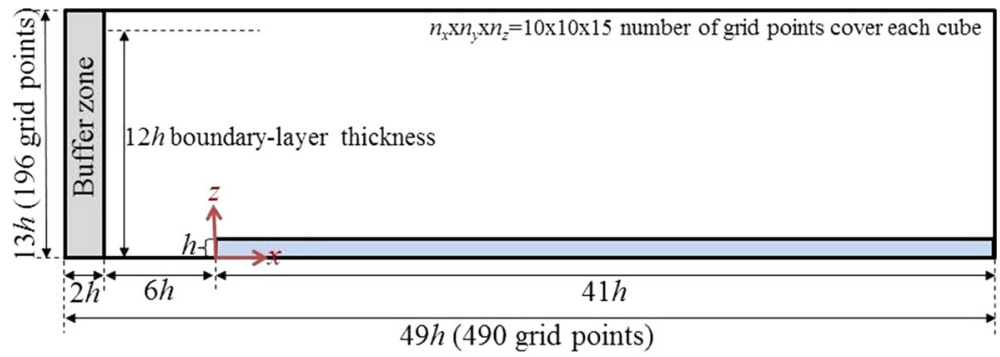

(b)

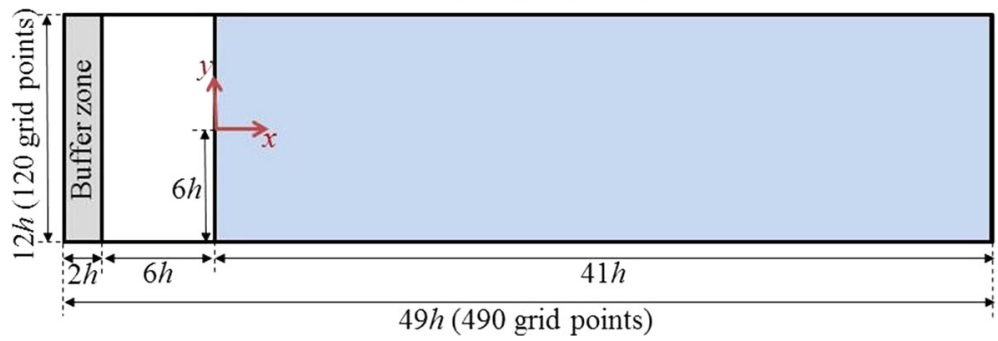

Fig. 5 Computational domain for the simulations of atmospheric boundary-layer flow past different urban-like surfaces: a cross-sectional view, and $\mathbf{b}$ top view

(a)
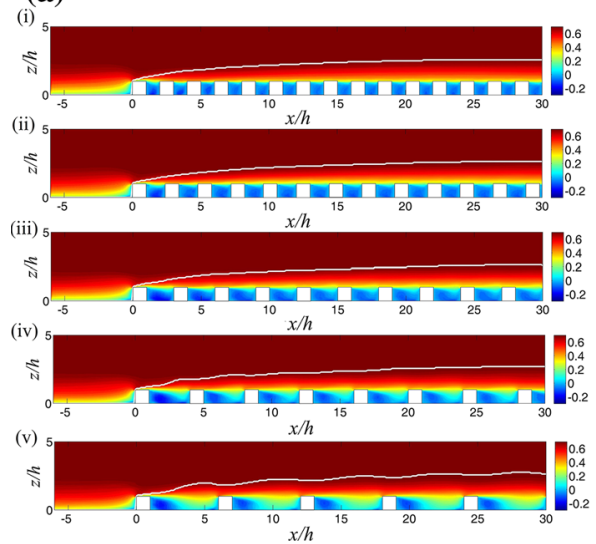

(b)
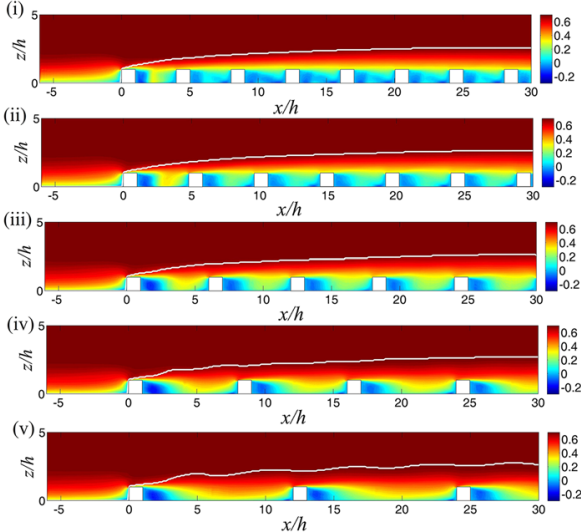

Fig. 6 Contours of $\langle\tilde{u}\rangle / U_{0}$ at centreline of the cube array and the edge of the IBL (white solid line) for a aligned array and $\mathbf{b}$ staggered array with $\lambda_{\mathrm{f}}=($ i) 0.25 , (ii) 0.174 , (iii) 0.111 , (iv) 0.063 and (v) 0.028

$(\approx 0.2 \delta)$ shortly downstream of the surface transition for all the cases. This is related to the higher building height relative to the ABL thickness considered in the current LES. For the cube arrays with lower densities, oscillating patterns of the IBL edges are identified as shown in Fig. 6. For these cases, there is a larger separation between cubes that provides more space for flow recovery before the flow passes the next row of cubes. This implies a higher wind speed just before the next row of cubes in these cases compared with the other cases with higher densities. This resulted in a larger speed-up of the flow just above the downstream cubes and, in turn, a slight decrease of the height of the IBL over the cubes.

For the flow within the array, in all the cases, the mean velocity is found to adjust quickly to the array as the shapes and magnitudes of the wakes are almost the same after the second 
(a)
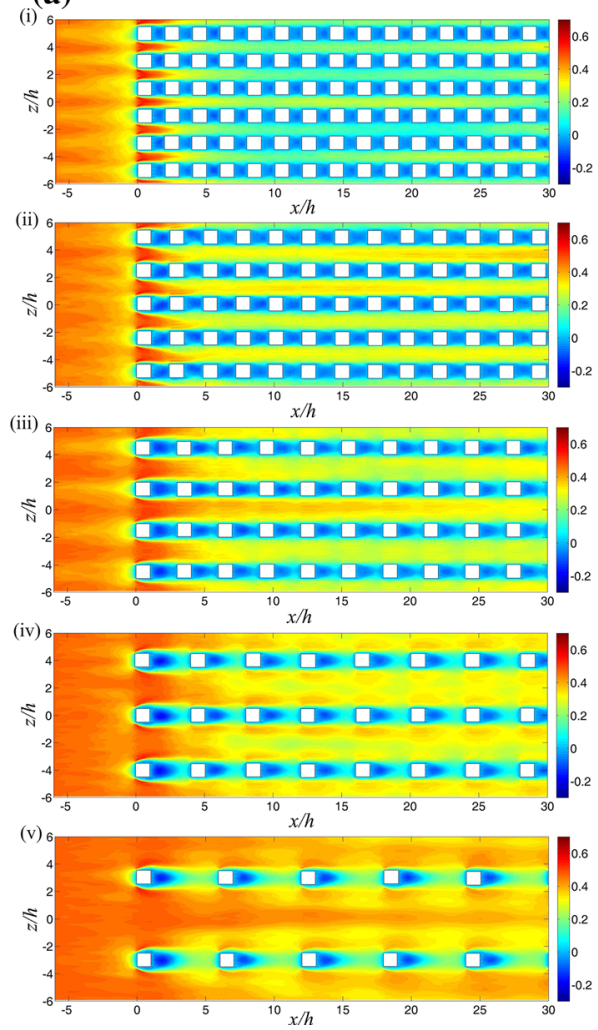

(b)
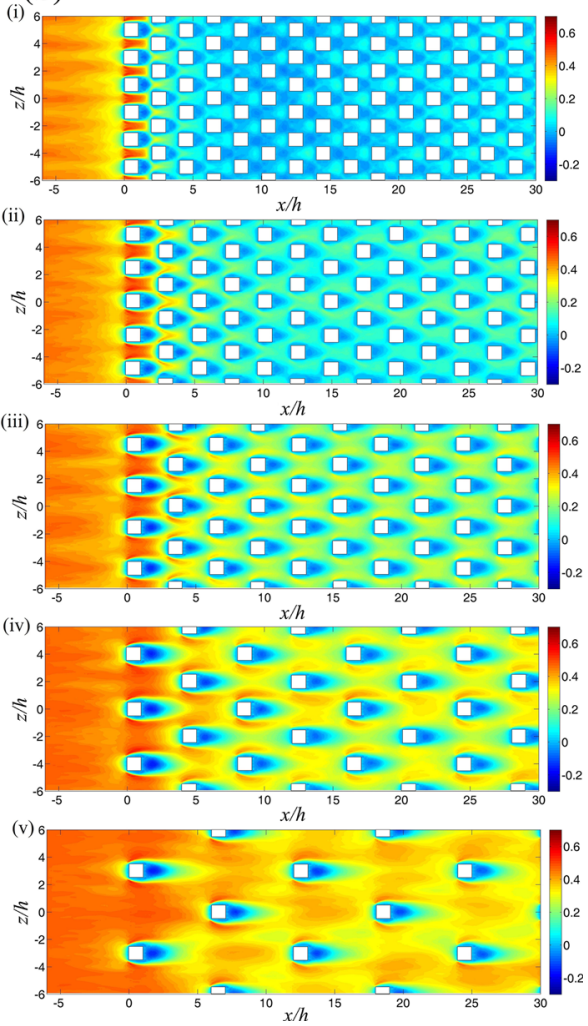

Fig. 7 Contours of $\langle\tilde{u}\rangle / U_{0}$ at $z=h / 2$ for a aligned array and $\mathbf{b}$ staggered array with $\lambda_{\mathrm{f}}=(i) 0.25$, (ii) 0.174 , (iii) 0.111 , (iv) 0.063 and (v) 0.028

row of cubes. The contours of the mean streamwise velocity component at the horizontal plane at $z=0.5 h$ for the different cases are also shown in Fig. 7. As expected, the wind speed within the array decreases with increasing density. A recirculation zone is formed behind each cube as shown by the regions of negative streamwise velocity component in Figs. 6 and 7. For the case of $\lambda=0.25$ for the aligned array, the region within the urban canopy is mostly occupied by flow recirculations and the flow within the canopy is almost completely separated from the boundary-layer flow above. This flow pattern is often classified as skimming flow in the literature (Perrier et al. 1972; Wieringa 1993; Pietri et al. 2009). On the other hand, for the case of $\lambda_{\mathrm{f}}=0.028$ for the staggered array, the flow within and above the cube array is similar to the semi-smooth turbulent flow conditions within and above a sparse canopy. In this case, the cubes are sufficiently far apart and their individual wakes are almost dissipated in the interspaces between the cubes. In this condition, the streamwise extent of the recirculation zone behind the cubes is found to be approximately $2 h$. For the other cases with intermediate cube densities, the flow is in the so-called wake-interference regime. In this regime, the separation between cubes is slightly less than individual wake lengths and the bulk flow above is able to penetrate into the upper region of the urban canopy.

Comparing the flow patterns at the downstream positions of cubes in the aligned and staggered arrays with the same density, a larger streamwise velocity component is found 
in the staggered arrays than in the aligned arrays. This is due to the fact that the staggered pattern induces the flow to change direction towards the regions downstream of the cubes as the flow passes through different rows of cubes. For the aligned arrays, the flow mainly passes through the channels between columns of cubes. In addition, the staggered pattern also corresponds to larger streamwise separation between cubes, which allows the individual wakes from each cube to recover more before encountering the next cube, as shown in Figs. 6 and 7.

\subsection{Turbulent Shear Stress and Streamwise Velocity Variance Patterns}

The contours of the total turbulent (Reynolds) shear stress $\left\langle u^{\prime} w^{\prime}\right\rangle\left(=\left\langle\tilde{u}^{\prime} \tilde{w}^{\prime}\right\rangle+\left\langle\tau_{x z}\right\rangle\right)$ at the cube-array centreline are shown in Fig. 8 for the different cases. Large negative turbulent stress is observed behind the cubes just above their top, indicating a maximum downward momentum flux associated with the entrainment of boundary-layer air into the wakes. As with the mean velocity and other turbulence statistics, the shear-stress is found to adjust quickly within the array, as a similar wake pattern is observed behind each cube after the second row of cubes. The configuration of the cubes in the array as well as the cube density are found to have an effect on the shear-stress distribution. For example, for the aligned array with the highest cube density $\left(\lambda_{\mathrm{f}}=0.25\right)$, a smaller overall $\left\langle u^{\prime} w^{\prime}\right\rangle$ magnitude is observed above the cubes compared with the other cases. This is related to the high cube density that hinders entrainment of boundary-layer air into the canopy. This is consistent with the discussion in Sect. 4.1 that the skimming flow regime exists and the flow within the canopy is almost completely separated from the bulk flow above. For the staggered case with the lowest density $\left(\lambda_{\mathrm{f}}=0.028\right)$, the streamwise distance between the cubes is the largest among all the cases and the magnitude of $\left\langle u^{\prime} w^{\prime}\right\rangle$ already decreases significantly before the flow passes the next downstream cube. This is consistent with the fact that, in this case, the cube array behaves like a sparse canopy in which the wakes of the cubes are almost dissipated in the interspaces between cubes as shown in Figs. 6 and 7.

The contours of the total streamwise velocity variance $\left\langle u^{\prime} u^{\prime}\right\rangle\left(=\left\langle\tilde{u}^{\prime} \tilde{u}^{\prime}\right\rangle+\left\langle\tau_{x x}\right\rangle\right)$ at the cube-array centreline for the different cases are shown in Fig. 9. Large velocity variance is found immediately above the first row of cubes for all the cases. Specifically, the maximum velocity variance is found at the same positions (behind the cubes just above $h$ ) as that of the maximum stress $\left(-\left\langle u^{\prime} w^{\prime}\right\rangle\right)$. This is because of the maximum turbulent stress and high shear there enhance the TKE production and therefore increase the velocity variance. Except for the aligned array with the highest cube density, a large velocity variance is observed behind every rows of cubes at a height of $z=h$. For the arrays with lower densities, the high velocity variance region extends to about $5 h$ downstream of the cubes.

\subsection{Drag and Drag Coefficients}

The drag exerted by buildings on ABL flow and the corresponding drag coefficients of the buildings are important quantities in the parametrization of flows within and above urban canopies (Raupach 1992; Macdonald et al. 1998; Macdonald 2000; Coceal and Belcher 2004). However, despite their importance, the changes of the drag force and drag coefficient with building configuration and density are still not well understood. Therefore, it is of interest to use the current LES to study the drag force and drag coefficients of the cubes in the aligned and staggered arrays with different densities.

Using the LES results, the drag exerted by the cubes on the flow $\left(D_{\mathrm{f}}\right)$ and the drag coefficient of the cubes $\left(C_{\mathrm{d}}\right)$ in the aligned and staggered arrays are calculated using the 
(a)
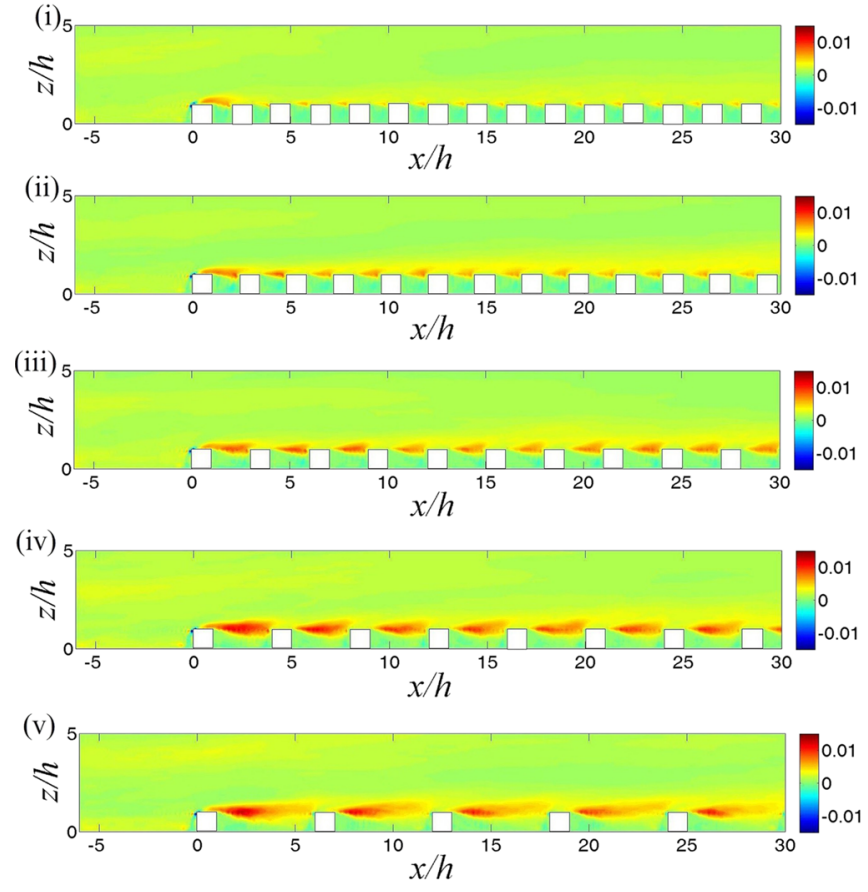

(b)
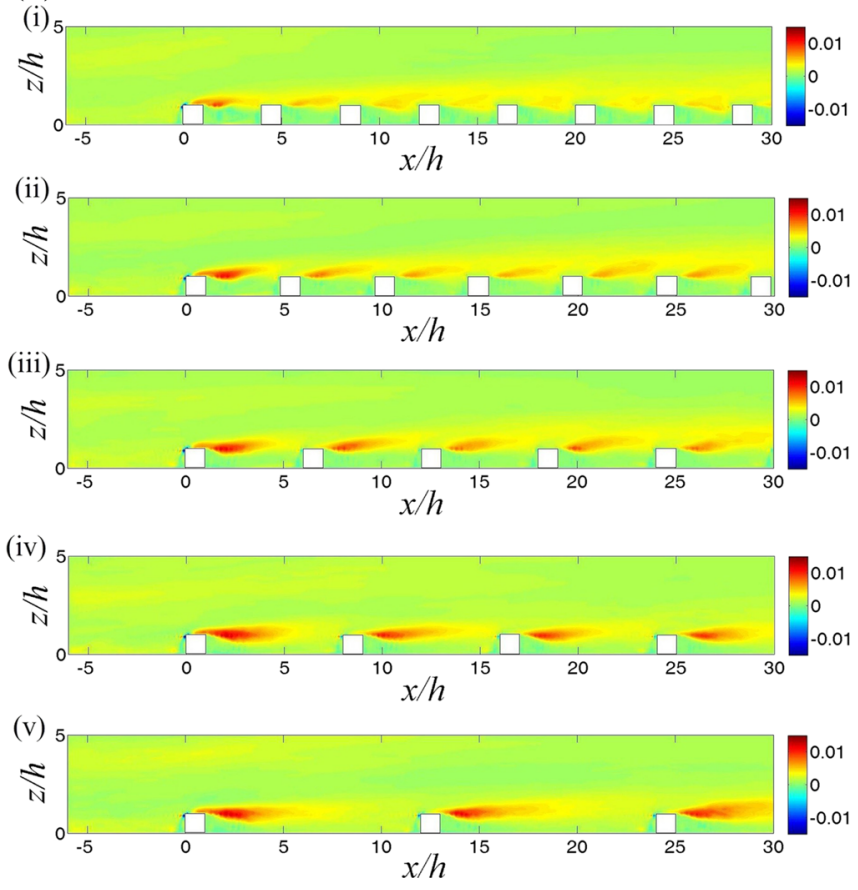

Fig. 8 Contours of $-\left\langle u^{\prime} w^{\prime}\right\rangle / U_{0}^{2}$ at the centreline of the cube array for $\mathbf{a}$ aligned array and $\mathbf{b}$ staggered array with $\lambda_{\mathrm{f}}=($ i) 0.25 , (ii) 0.174, (iii) 0.111 , (iv) 0.063 and (v) 0.028 

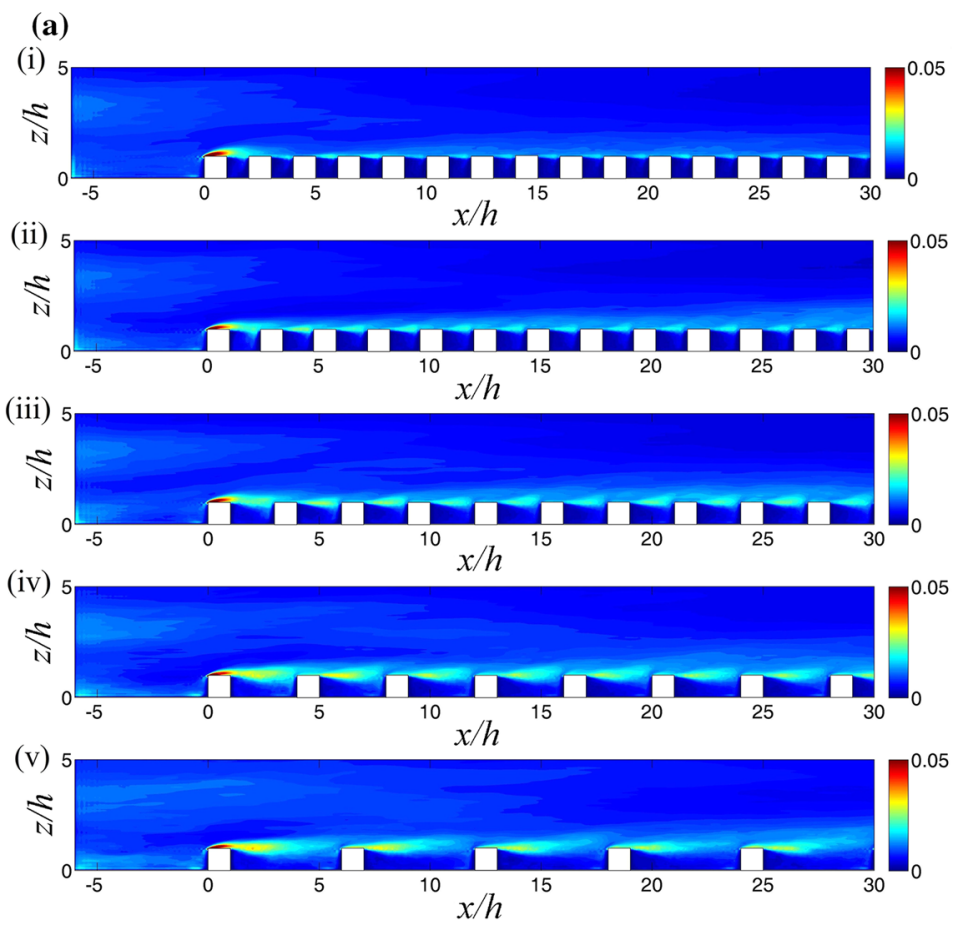

(b)
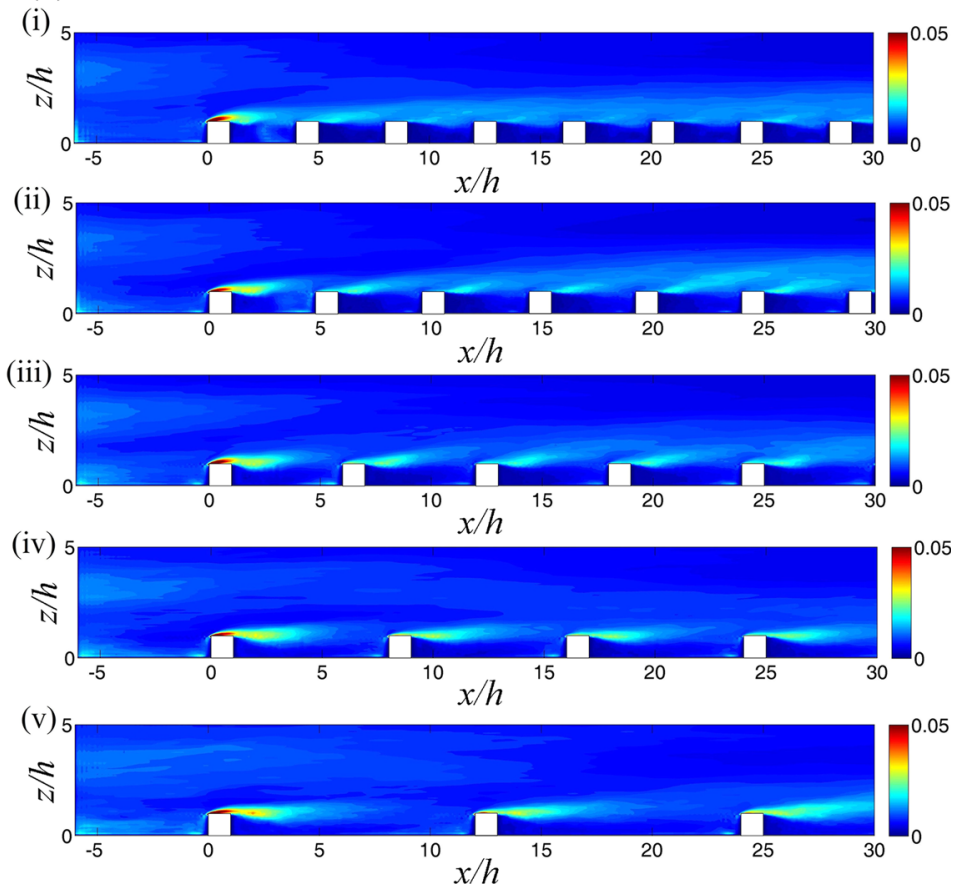

Fig. 9 Contours of $\left\langle u^{\prime} u^{\prime}\right\rangle / U_{0}^{2}$ at the centreline of the cube array for a aligned array and $\mathbf{b}$ staggered array with $\lambda_{\mathrm{f}}=($ i) 0.25 , (ii) 0.174 , (iii) 0.111 , (iv) 0.063 and (v) 0.028 
Fig. 10 Examples of a repeating cube unit and a repeating row unit in aligned and staggered arrays

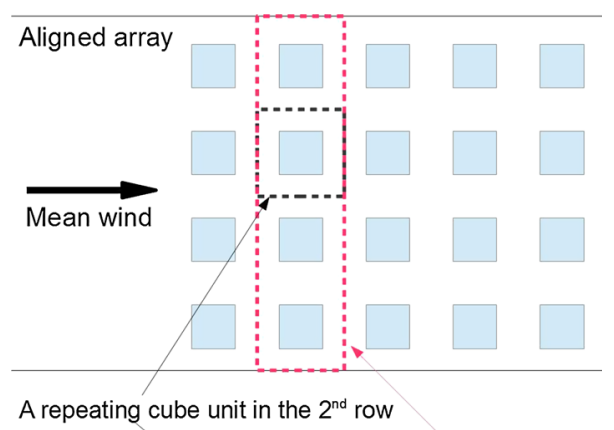

A repeating row unit (the $2^{\text {nd }}$ row)

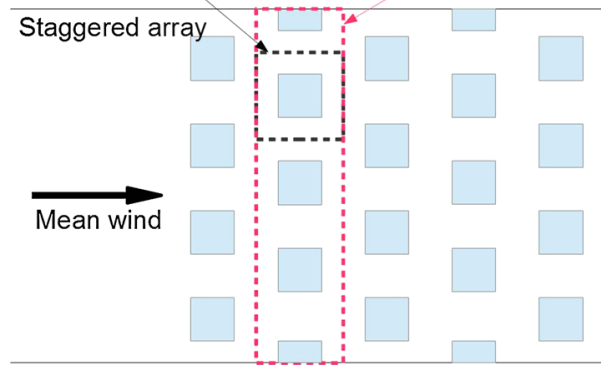

(a)
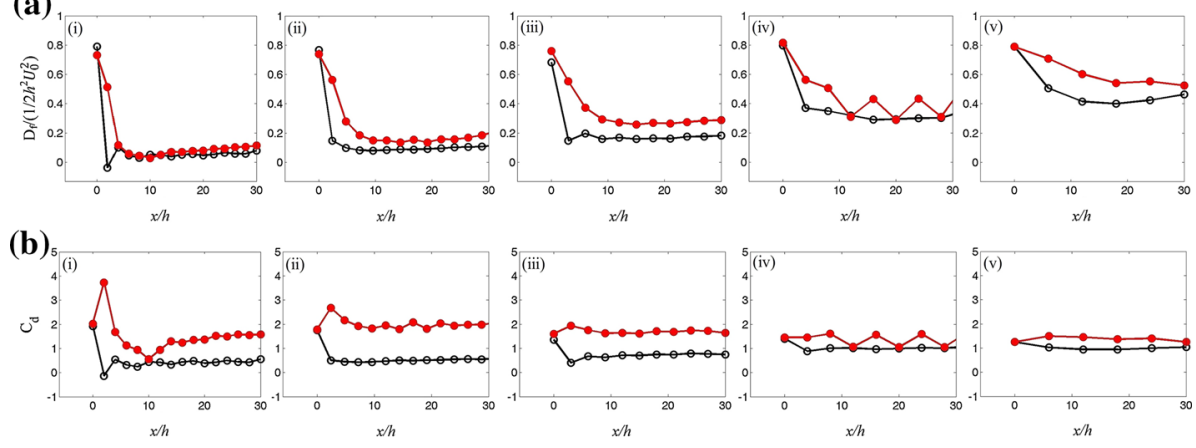

Fig. 11 a Normalized drag force and $\mathbf{b}$ drag coefficient of cubes on different rows in the aligned (black line and hollow circle) and staggered arrays (red line and filled circle) with $\lambda_{\mathrm{f}}=$ (i) 0.25 , (ii) 0.174 , (iii) 0.111 , (iv) 0.063 and (v) 0.028

pressure difference $(\Delta p)$ between the upwind and downwind surfaces of the cube as

$$
\begin{aligned}
& D_{\mathrm{f}}=\langle\Delta p\rangle_{s} h^{2}, \\
& C_{\mathrm{d}}=2\langle\Delta p\rangle_{s} /\left\langle\tilde{u}^{2}\right\rangle_{\text {vol }} .
\end{aligned}
$$

Here $\langle\Delta p\rangle_{s}$ is the temporally- and spatially- averaged value of $\Delta p$ (over the frontal surface of the cube), and $\left\langle u^{2}\right\rangle_{\mathrm{vol}}$ represents the temporally- and volume spatially-averaged value [over one repeating cube unit (see Fig. 10)] of the squared streamwise velocity component within the canopy $(z \leq h)$.

For all the cases, larger values of $D_{\mathrm{f}}$ are found for the first row of cubes (with normalized values $\approx 0.7-0.8$ ) as shown in Fig. 11. In order to compare drag for different values of $U_{0}$, 
$D_{\mathrm{f}}$ is normalized by $\frac{1}{2} h^{2} U_{0}^{2}$ to give a dimensionless drag force. In most cases, the values of $D_{\mathrm{f}}$ attain an approximate constant value after the second or third row of cubes, consistent with rapid flow adjustment. After the third row, increases in the values of $D_{\mathrm{f}}$ with decreasing density are found for both the aligned and staggered arrays. The normalized adjusted values of $D_{\mathrm{f}}$ for the cubes in the staggered arrays increase from about 0.1 to 0.5 as $\lambda_{\mathrm{f}}$ decreases from 0.25 to 0.028 . These are larger than those of the aligned arrays in which the normalized adjusted values of $D_{\mathrm{f}}$ increase from about 0.1 to 0.4 as $\lambda_{\mathrm{f}}$ decreases from 0.25 to 0.028 . This is related to the stronger sheltering effects of the upstream cubes for the aligned arrays compared with the staggered cases.

For the values of $C_{\mathrm{d}}$ for the cubes in the staggered arrays, the maximum value is found at the second row of cubes for all the cases except that of $\lambda_{\mathrm{f}}=0.063$ (with values ranging from about 1.5 to 4 ). The peak value of $C_{\mathrm{d}}$ found at the second row of cubes is related to the staggered pattern of the cubes and the flow speed-up near the first row of cubes due to the blockage of the flow by the first row of cubes. The flow speed-up is larger for the arrays with higher density. For the array with the highest density tested $\left(\lambda_{\mathrm{f}}=0.25\right)$, the value of $C_{\mathrm{d}}$ for the cubes at the second rows attains a value $\approx 4$ that is the largest among all the staggered cases. It is also interesting that cubes in the first and third rows of a staggered array have similar values of $C_{\mathrm{d}}$. In contrast, for the aligned arrays, smaller values of $C_{\mathrm{d}}$ are found for the downstream cubes compared with the first row of cubes. Larger values of $C_{\mathrm{d}}$ are observed for the downstream cubes of the staggered arrays (with values ranging from 1.5 to 2 ) than the aligned arrays (with values ranging from 0.5 to 1 ). The differences are larger for the arrays with higher density $\left(\lambda_{\mathrm{f}}=0.25,0.174\right.$ and 0.111$)$. For the aligned arrays with these densities, the values of $C_{\mathrm{d}}$ become very small with values of about 0.5 .

\subsection{Effective Roughness Length and Displacement Height}

To obtain the effective roughness length $\left(z_{0}\right)$ and displacement height $(d)$ of the cube arrays using the simulation results, the mean velocity and turbulent shear-stress fields are streamwise and spanwise spatially-averaged over one repeating row unit of a row (see Fig. 10) located inside the range of $21<x / h<30$. The repeating unit chosen in this condition is at least $21 \mathrm{~h}$ downstream of the surface transition and $11 \mathrm{~h}$ upstream of the end of the domain. The choice of this range is to allow sufficient space for the flow to adjust to the surfaces and to avoid the influence of the buffer zone on the flow. For the different cases, the maximum value of $-\left\langle u^{\prime} w^{\prime}\right\rangle$ in the streamwise and spanwise averaged profile near $z=h$ is used to estimate the friction velocity $u_{*}$ of the surfaces. The values of $z_{0}$ and $d$ are then obtained by fitting a logarithmic height function (Eq. 1 with $\kappa=0.4$ ) to the wind profiles just above the cube arrays.

The results for $z_{0}$ and $d$ are shown in Fig. 12 together with the theoretical expressions of Macdonald et al. (1998) and Raupach (1992), and the wind-tunnel experimental results of Cheng et al. (2007). The current results show that the values of $d$ for the aligned and staggered arrays have roughly the same pattern and magnitude as a function of $\lambda_{\mathrm{f}}$. In particular, the values of $d / h$ are found to increase from 0.65 to 0.9 as $\lambda_{\mathrm{f}}$ increases from 0.028 to 0.25 . For $z_{0}$, larger values are found for the staggered arrays than those of the aligned arrays with the same density. For the staggered arrays, $z_{0}$ increases from $0.02 h$ to $0.05 h$ as $\lambda_{\mathrm{f}}$ increases from 0.028 to 0.111 and then decreases from $0.05 h$ to $0.03 h$ as $\lambda_{\mathrm{f}}$ further increases from 0.174 to 0.25 . For the aligned arrays, $z_{0}$ increases from $0.017 h$ to $0.023 h$ as $\lambda_{\mathrm{f}}$ increases from 0.028 to 0.174 and then decreases to a value of about $0.016 h$ as $\lambda_{\mathrm{f}}$ further increases from 0.174 to 0.25 . The results generally agree with the wind-tunnel experimental results of Cheng et al. (2007) as similar magnitudes of $z_{0}$ and $d$ are found. For the theories of $z_{0}$ proposed in 

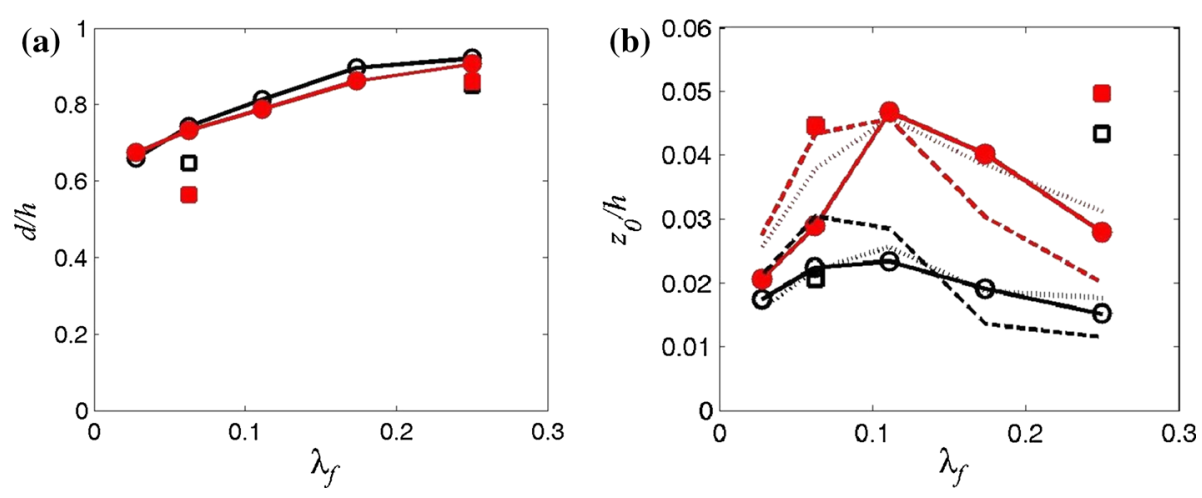

Fig. 12 Normalized displacement height (a) and surface roughness (b) as a function of $\lambda_{\mathrm{p}}$ for aligned and staggered arrays. Black line and hollow circle aligned array, current LES; red line and filled circle staggered array, current LES; black hollow square aligned array, Cheng et al. (2007); red filled square staggered array, Cheng et al. (2007); black dashed line Macdonald et al. (1998), $C_{\mathrm{d}}=2.2$; red dashed line Macdonald et al. (1998), $C_{\mathrm{d}}=2.9$; black dotted line Raupach (1992), $C_{\mathrm{R}}=0.4$; red dotted line Raupach (1992), $C_{\mathrm{R}}=0.65$

Macdonald et al. (1998) and Raupach (1992), there is a parameter (denoted by $C_{\mathrm{D}}$ and $C_{\mathrm{R}}$ in Macdonald et al. (1998) and Raupach (1992), respectively) that can be interpreted as the normalized effective drag coefficient of a cube immersed in an array. Here, in particular, the values of $C_{\mathrm{D}}=2.9(2.2)$ and $C_{\mathrm{R}}=0.65(0.4)$ are used for the staggered (aligned) arrays in the expressions of Macdonald et al. (1998) and Raupach (1992), respectively. These results are consistent with the discussion in Sect. 4.3 that larger values of drag coefficients for the cubes in the staggered arrays than those of the aligned arrays are found. Excellent agreement is observed between the simulation results and the analytical expressions of Raupach (1992). In particular, the changes of $z_{0}$ with density for the aligned and staggered arrays are well predicted. The LES results also agree fairly well with the expressions of Macdonald et al. (1998) although slight differences are observed.

Note that in the theoretical expressions for $z_{0}$ of Raupach (1992) and Macdonald et al. (1998), the values of $d$ are needed as an input parameter. Here the fitted values of $d$ from the current simulation results are used. Also, the other parameters $\left(c_{1}, c_{2}\right.$ and $c_{w}$ in Eqs. 18 and 30 of Raupach (1992)) in the expression of Raupach (1992) have been set to the values used by Raupach (1992) $\left(c_{1}=c_{2}=0.5\right.$ and $\left.c_{w}=1.5\right)$.

\subsection{Effective Mixing Length}

The Prandtl mixing-length model is commonly used to parametrize the temporally- and horizontal spatially-averaged turbulent shear stress $\left(\left\langle u^{\prime} w^{\prime}\right\rangle_{\text {hor }}\right)$ in Regnolds-averaged NavierStokes and 1-D models of urban canopy flows (Macdonald 2000; Coceal and Belcher 2004). Specifically, the model can be written as,

$$
\left\langle u^{\prime} w^{\prime}\right\rangle_{\text {hor }}=-l_{\mathrm{m}}(z)^{2}\left|\frac{\partial\langle u\rangle_{\text {hor }}}{\partial z}\right| \frac{\partial\langle u\rangle_{\text {hor }}}{\partial z}
$$

where $\langle u\rangle_{\text {hor }}$ denotes the temporally- and horizontal spatially-averaged streamwise velocity component and $l_{\mathrm{m}}$ is the effective mixing length. In his urban canopy model, Macdonald (2000) considered $l_{\mathrm{m}}$ to be uniform with height inside urban canopies, having the disadvantage that the no-slip condition at the ground is not satisfied. Later, instead of a constant value inside 
(a)
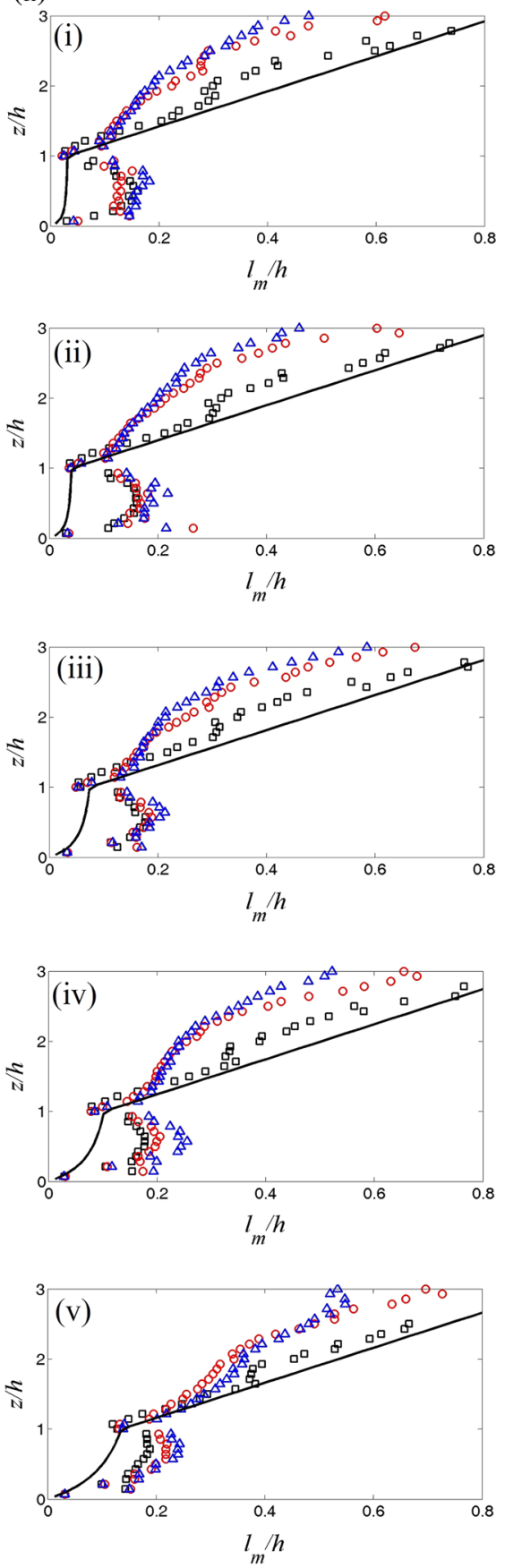

(b)
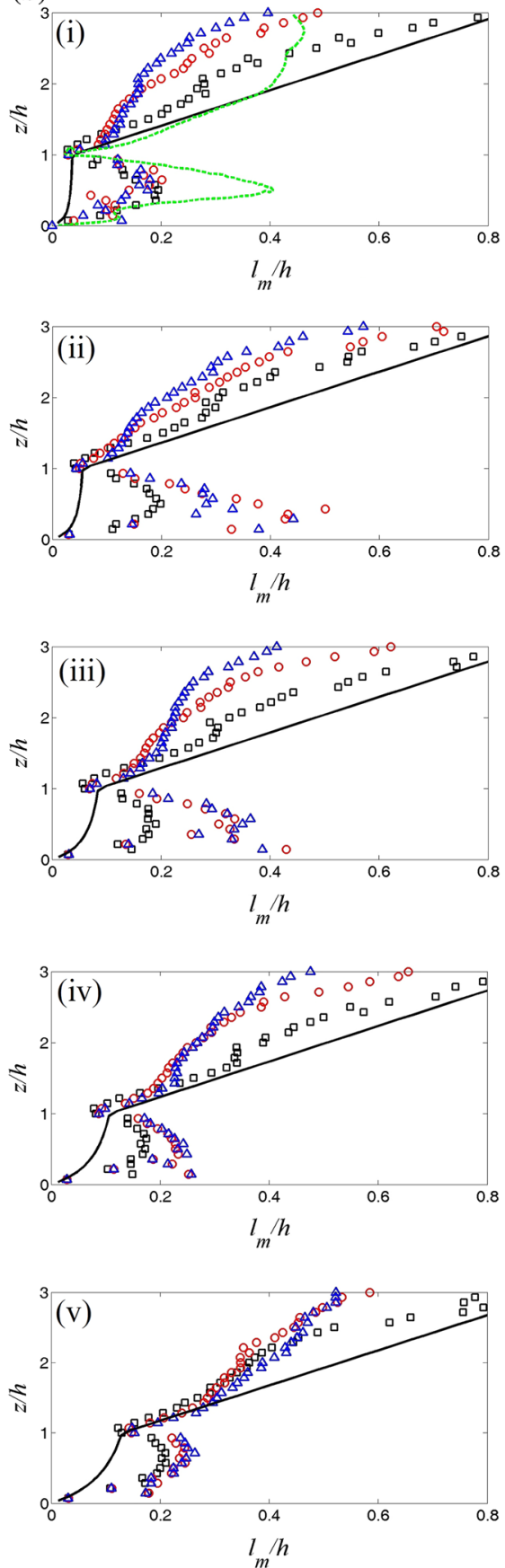

Fig. 13 Profiles of effective mixing length of $\mathbf{a}$ aligned and $\mathbf{b}$ staggered arrays with $\lambda_{\mathrm{f}}=$ (i) 0.25 , (ii) 0.174 , (iii) 0.111, (iv) 0.063 and (v) 0.028. Black square first row of cubes, current LES; red circle third row of cubes, current LES; blue triangle fifth row of cubes; black line model of Coceal and Belcher (2004); green dashed line DNS of Coceal et al. (2006) 
urban canopies, Coceal and Belcher (2004) suggested the following expression for $l_{\mathrm{m}}$

$$
\frac{1}{l_{\mathrm{m}}(z)}=\frac{1}{\kappa z}+\frac{1}{l_{\mathrm{c}}}
$$

with $l_{\mathrm{c}}$ computed by fixing $l_{\mathrm{m}}(z=h)=\kappa(h-d)$, i.e.,

$$
\frac{1}{\kappa(h-d)}=\frac{1}{\kappa h}+\frac{1}{l_{\mathrm{c}}}
$$

Using this model, the no-slip condition at the ground is satisfied. In view of the limited amount of information on $l_{\mathrm{m}}$ profiles within urban canopies, here we calculate $l_{\mathrm{m}}$ within and above the cube arrays using the LES results according to Eq. 11 in which the horizontal spatial average is taken over different repeating row units (see Fig. 10). Here, in the spatial averaging, the volumes occupied by the cubes in the arrays are excluded.

The results are shown and compared with the model of Coceal and Belcher (2004) in Fig. 13. Note that for this model (Eqs. 12 and 13), the value of $d$ is needed as an input parameter and the values obtained from the analysis presented in Sect. 4.4 are used. In comparison, the values of $l_{\mathrm{m}}$ in the urban canopies calculated from the current LES results are significantly larger than those predicted by the model of Coceal and Belcher (2004). In particular, this model predicts an increase of $l_{\mathrm{m}}$ with height inside the urban canopy, while a local maximum of $l_{\mathrm{m}}$ is found within the canopy for all the LES cases. The local maximum of $l_{\mathrm{m}}$ within the canopy is related to the significant decrease of the mean velocity gradient within the canopy, while relatively high turbulence level is still found there. The LES results show similar behaviour to the DNS study of Coceal et al. (2006) for a staggered array of cubes with $\lambda_{\mathrm{f}}=0.25$ (Fig. 13b-i). In contrast, at canopy roof level, a sharp change in velocity with height is found for all the cases, resulting in a large velocity gradient and a small value of $l_{\mathrm{m}}$ there.

By comparing the profiles of $l_{\mathrm{m}}$ for different rows, it is found that, within the canopy, $l_{\mathrm{m}}$ adjusts quickly to the canopy and shows a similar pattern after the second or third row of cubes for most of the cases. This is consistent with the results of rapid adjustment of the mean flow and turbulence discussed in Sect. 4.1. Smaller values of $l_{\mathrm{m}}$ are generally found for the first row compared with those of the downstream rows for all the cases. This is related to the larger vertical velocity gradient near the first row of cubes (see Fig. 3). For the region just above the canopy, flow adjustment can also be observed since the values of $l_{\mathrm{m}}$ agree well with the prediction of $l_{\mathrm{m}}=\kappa(z-d)$. These results also justify the displacement-height estimation method used in Sect. 4.4.

For the aligned arrays of different densities, the magnitudes of $l_{\mathrm{m}}$ are approximately the same and equal to $0.2 h$ in the middle of the canopies. Near the ground and the top of the canopies, smaller values of $l_{\mathrm{m}}$ are found. For the staggered arrays with $\lambda_{\mathrm{f}}=0.25,0.063$, and $0.028, l_{\mathrm{m}}$ values are found to be more or less the same as those of the aligned cases with magnitudes of about $0.2 h$ in the middle of the canopies. For the cases of $\lambda_{f}=0.174$, and 0.111 , larger magnitudes of $l_{\mathrm{m}}$ are found for the staggered arrays with values of about $0.4 \mathrm{~h}$ at $z=h / 2$.

\section{Conclusions}

Large-eddy simulations (LES) were performed to investigate the adjustment of a turbulent boundary-layer flow to idealized urban surfaces represented by uniform arrays of cubes. The LES code combined the modulated gradient subgrid-scale model (Lu and Porté-Agel 2010) 
and an immersed boundary method to simulate the presence of the cubes. The method was validated in Cheng and Porté-Agel (2013) in the simulation of a flow past a two-dimensional surface mounted block, and it is further validated here with the wind-tunnel experimental data of Brown et al. (2001) for a boundary-layer flow past an aligned array of cubes. Good agreement between the experimental data and the LES results are observed in the vertical and spanwise profiles of the mean velocity and velocity variances at different streamwise locations.

The LES framework is then used to simulate atmospheric boundary-layer (ABL) flow over a surface transition from a flat homogeneous terrain to uniform arrays of cubes. Aligned and staggered arrays with five different frontal area densities $\left(\lambda_{f}\right)$, equal to $0.028,0.063$, $0.111,0.174$ and 0.250 , are considered. The simulation results show the development of an internal boundary layer (IBL) over the arrays of cubes. No significant difference in the depth of the IBL among the different cases is found. For the region within the arrays, different flow patterns are observed for different building configurations and densities. Both the mean wind and turbulence are found to adjust quickly after the surface transition.

In order to provide information for the characterization and modelling of flows within and above urban canopies, the drag exerted by the cubes on the flow $\left(D_{\mathrm{f}}\right)$ and the drag coefficients of the cubes $\left(C_{\mathrm{d}}\right)$ are calculated explicitly using the LES results. Consistent with the rapid flow adjustment downstream of the surface transition, the value of $D_{\mathrm{f}}$, in most of the cases, attains an approximately constant value after the second or third row of cubes. For those adjusted values, values of both $D_{\mathrm{f}}$ and $C_{\mathrm{d}}$ are found to be larger for the staggered arrays than for the aligned arrays with the same $\lambda_{\mathrm{f}}$. At a downstream location where the flow immediately above the cube array is already adjusted to the surface, the spatially-averaged streamwise velocity component is found to have a logarithmic profile for all the cases. For both the aligned and staggered configurations, the values of the displacement height $d$ are found to increase roughly from $0.65 h$ to $0.9 h$ as $\lambda_{\mathrm{f}}$ increases from 0.028 to 0.25 . For the aerodynamic roughness length, $z_{0}$, a maximum value at $\lambda_{\mathrm{f}}=0.11$ is observed for both the aligned and staggered arrays, and larger values of $z_{0}$ are obtained for the staggered array than for the aligned one with the same density. The results for $z_{0}$ are found to agree well with the theoretical expressions of Raupach (1992) using a larger effective drag coefficient for the staggered arrays than the aligned cases. This is consistent with the results that larger values of $C_{\mathrm{d}}$ are found for the staggered arrays than those of the aligned arrays with the same $\lambda_{\mathrm{f}}$. The values of the effective mixing length $\left(l_{\mathrm{m}}\right)$ within and above the aligned and staggered arrays of different densities are calculated as a function of height using the LES results. In contrast to the previous model of Coceal and Belcher (2004), a local maximum of $l_{\mathrm{m}}$ within the canopy is found in all the cases, with values ranging from $0.2 h$ to $0.4 h$.

The results presented herein should help improve and extend the current modelling methods for flow and turbulence within and above urban canopies, especially at the rural-to-urban flow transition region where the flow adjustment behaviour is less investigated in the literature. Future work will continue the development of the LES framework to simulate the transport of active and passive scalars within and above urban canopies, and to further investigate the effects of wind direction, non-uniform building heights, and thermal stability.

Acknowledgments We gratefully acknowledge Michael J. Brown for kindly supplying the wind-tunnel data for our LES validation. This research was supported by the Swiss National Science Foundation (grant 200021132122 and IZERZ0-142168) and the Swiss Innovation and Technology Committee (CTI) within the context of the Swiss Competence Center for Energy Research 'FURIES: Future Swiss Electrical Infrastructure'. Computing resources were provided by the Swiss National Supercomputing Centre (CSCS) under project ID s467. 


\section{References}

Abkar M, Porté-Agel F (2012) A new boundary condition for large-eddy simulation of boundary-layer flow over surface roughness transitions. J Turbul 13:1-18

Albertson JD, Parlange MB (1999) Surface length scales and shear stress: implications for land-atmosphere interaction over complex terrain. Water Resour Res 35:2121-2132

Belcher SE, Jerram N, Hunt JCR (2003) Adjustment of a turbulent boundary layer to a canopy of roughness elements. J Fluid Mech 488:369-398

Boppana VBL, Xie Z-T, Castro IP (2010) Large-eddy simulation of dispersion from surface sources in arrays of obstacles. Boundary-Layer Meteorol 135:433-454

Brown MJ, Lawson RE, DeCroix DS, Lee RL (2001) Comparison of centerline velocity measurements obtained around $2 \mathrm{D}$ and $3 \mathrm{D}$ building arrays in a wind tunnel. Tech. Rep, Los Alamos National Laboratory, NM, $7 \mathrm{pp}$

Businger JA, Wyngaard JC, Izumi Y, Bradley EF (1971) Flux-profile relationships in the atmospheric surface layer. J Atmos Sci 28:181-189

Canuto C, Hussaini MY, Quarteroni AM, Zang TA (1988) Spectral methods in fluid dynamics. Springer, New York $568 \mathrm{pp}$

Castillo MC, Inagaki A, Kanda M (2011) The effects of inner-and outer-layer turbulence in a convective boundary layer on the near-neutral inertial sublayer over an urban-like surface. Boundary-Layer Meteorol 140:453-469

Castro IP, Cheng H, Reynolds R (2006) Turbulence over urban-type roughness: deductions from wind-tunnel measurements. Boundary-Layer Meteorol 118:109-131

Chamorro LP, Porté-Agel F (2010) Wind-tunnel study of surface boundary conditions for large-eddy simulation of turbulent flow past a rough-to-smooth surface transition. J Turbul 11:1-17

Cheng H, Castro IP (2002a) Near-wall flow development after a step change in surface roughness. BoundaryLayer Meteorol 105:411-432

Cheng H, Castro IP (2002b) Near wall flow over urban-like roughness. Boundary-Layer Meteorol 104:229-259

Cheng H, Hayden P, Robins AG, Castro IP (2007) Flow over cube arrays of different packing densities. J Wind Eng Ind Aerodyn 95:715-740

Cheng W-C, Porté-Agel F (2013) Evaluation of subgrid-scale models in large-eddy simulation of flow past a two-dimensional block. Int J Heat Fluid Flow 44:301-311

Coceal O, Belcher SE (2004) A canopy model of mean winds through urban areas. Q J R Meteorol Soc 130:1349-1372

Coceal O, Belcher SE (2005) Mean winds through an inhomogeneous urban canopy. Boundary-Layer Meteorol 115:47-68

Coceal O, Thomas TG, Castro IP, Belcher SE (2006) Mean flow and turbulence statistics over groups of urban-like cubical obstacles. Boundary-Layer Meteorol 121:491-519

Coceal O, Dobre A, Thomas TG, Belcher SE (2007a) Structure of turbulent flow over regular arrays of cubical roughness. J Fluid Mech 589:375-409

Coceal O, Thomas TG, Belcher SE (2007b) Spatial variability of flow statistics within regular building arrays. Boundary-Layer Meteorol 125:537-552

Fang J, Diebold M, Higgins C, Parlange MB (2011) Towards oscillation-free implementation of the immersed boundary method with spectral-like methods. J Comput Phys 230:8179-8191

Grimmond CSB, Oke TR (1999) Aerodynamic properties of urban areas derived from analysis of surface form. J Appl Meteorol 38:1262-1292

Hinze JO (1975) Turbulence, 2nd edn. McGraw-Hill, New York, 790 pp

Kanda M (2006) Large-eddy simulations on the effects of surface geometry of building arrays on turbulent organized structures. Boundary-Layer Meteorol 118:151-168

Lu H, Porté-Agel F (2010) A modulated gradient model for large-eddy simulation: application to a neutral atmospheric boundary layer. Phys Fluids 22:015109

Macdonald RW (2000) Modelling the mean velocity profile in the urban canopy layer. Boundary-Layer Meteorol 97:25-45

Macdonald RW, Griffiths RF, Hall DJ (1998) An improved method for the estimation of surface roughness of obstacle arrays. Atmos Environ 32:1857-1864

Macdonald RW, Carter Schofield S, Slawson PR (2002) Physical modelling of urban roughness using arrays of regular roughness elements. Water Air Soil Pollut 2:541-554

Marusic I, Kunkel GJ, Porté-Agel F (2001) Experimental study of wall boundary conditions for large-eddy simulation. J Fluid Mech 446:309-320

Meneveau C, Katz J (2000) Scale-invariance and turbulence models for large-eddy simulation. Annu Rev Fluid Mech 32:1-32 
Mittal R, Iaccarino G (2005) Immersed boundary methods. Annu Rev Fluid Mech 37:239-261

Orszag SA, Pao Y-H (1975) Numerical computation of turbulent shear flows. Adv Geophys 18 A:225-236

Pendergrass W, Aria SPS (1984) Dispersion in neutral boundary layer over a step change in surface roughnessI. Mean flow and turbulence structure. Atmos Environ 18:1267-1279

Perrier ER, Robertson JM, Millington RJ, Peter DB (1972) Spatial and temporal variation of wind above and within a soybean canopy. Agric Meteorol 10:421-442

Petersen RL (1997) A wind tunnel evaluation of methods for estimating surface roughness length at industral facilities. Atmos Environ 31(1):45-57

Pietri L, Petroff A, Amielh M, Anselmet F (2009) Turbulence characteristics within sparse and dense canopies. Environ Fluid Mech 9:297-320

Pope SB (2000) Turbulent flows. Cambridge University Press, Cambridge, $771 \mathrm{pp}$

Porté-Agel F (2004) A scale-dependent dynamic model for scalar transport in large-eddy simulations of the atmospheric boundary layer. Boundary-Layer Meteorol 112:81-105

Porté-Agel F, Meneveau C, Parlange MB (2000) A scale-dependent dynamic model for large-eddy simulation: application to a neutral atmospheric boundary layer. J Fluid Mech 415:261-284

Porté-Agel F, Wu YT, Lu H, Conzemius RJ (2011) Large-eddy simulation of atmospheric boundary layer flow through wind turbunes and wind farms. J Wind Eng Ind Aerodyn 99:154-168

Porté-Agel F, Wu YT, Chen CH (2013) A numerical study of the effects of wind direction on turbine wakes and power losses in a large wind farm. Energies 6:5297-5313

Raupach MR (1992) Drag and drag partition on rough surfaces. Boundary-Layer Meteorol 60:375-395

Raupach MR (1994) Simplified expressions for vegetation roughness length and zero-plane displacement as functions of canopy height and area index. Boundary-Layer Meteorol 71:211-216

Raupach MR, Thom AS, Edwards I (1980) A wind-tunnel study of turbulent flow close to regularly arrayed rough surfaces. Boundary-Layer Meteorol 18:373-397

Sagaut P (2006) Large eddy simulation for incompressible flows: an introduction, 3rd edn. Springer, Berlin, $558 \mathrm{pp}$

Savelyev SA, Taylor PA (2005) Internal boundary layers: I. Height formulae for neutral and diabatic flows. Boundary-Layer Meteorol 115:1-25

Schultz M, Schatzmann M, Leitl B (2005) Effect of roughness inhomogeneities on the development of the urban boundary layer. Int J Environ Pollut 25:105-117

Stoll R, Porté-Agel F (2006) Effect of roughness on surface boundary conditions for large-eddy simulation. Boundary-Layer Meteorol 118:169-187

Stoll R, Porté-Agel F (2008) Large-eddy simulation of the stable atmospheric boundary layer using dynamic models with different averaging schemes. Boundary-Layer Meteorol 126:1-28

Tseng Y-H, Meneveau C, Parlange MB (2006) Modeling flow around bluff bodies and predicting urban dispersion using large eddy simulation. Environ Sci Technol 40:2653-2662

Voller VR, Porté-Agel F (2002) Moore's law and numerical modeling. J Comput Phys 179:698-703

Wan F, Porté-Agel F (2011) Large-eddy simulation of stably-stratified flow over a steep hill. Boundary-Layer Meteorol 138(3):367-384

Wieringa J (1993) Representative roughness parameters for homogeneous terrain. Boundary-Layer Meteorol 63:323-363

Wood DH (1982) Internal boundary-layer growth following a step change in surface roughness. BoundaryLayer Meteorol 22:241-244

Wu YT, Porté-Agel F (2011) Large-eddy simulation of wind-turbine wakes: evaluation of turbine parametrisations. Boundary-Layer Meteorol 138:345-366

Wu YT, Porté-Agel F (2012) Atmospheric turbulence effects on wind-turbine wakes: an LES study. Energies 5:5340-5362

Wu YT, Porté-Agel F (2013) Simulation of turbulent flow inside and above wind farms: model validation and layout effects. Boundary-Layer Meteorol 146:181-205

Xie Z-T, Coceal O, Castro IP (2008) Large-eddy simulation of flows over random urban-like obstacles. Boundary-Layer Meteorol 129:1-23 